\title{
Temporal Bone CT: Improved Image Quality and Potential for Decreased Radiation Dose Using an Ultra-High-Resolution Scan Mode with an Iterative Reconstruction Algorithm
}

\author{
S. Leng, F.E. Diehn, J.I. Lane, K.K. Koeller, R.J. Witte, R.E. Carter, and C.H. McCollough
}

\begin{abstract}
BACKGROUND AND PURPOSE: Radiation dose in temporal bone CT imaging can be high due to the requirement of high spatial resolution. In this study, we assessed whether $\mathrm{CT}$ imaging of the temporal bone by using an ultra-high-resolution scan mode combined with iterative reconstruction provides higher spatial resolution and lower image noise than a z-axis ultra-high-resolution mode.
\end{abstract}

MATERIALS AND METHODS: Patients with baseline temporal bone CT scans acquired by using a z-axis ultra-high-resolution protocol and a follow-up scan by using the ultra-high-resolution-iterative reconstruction technique were identified. Images of left and right temporal bones were reconstructed in the axial, coronal, and Poschl planes. Three neuroradiologists assessed the spatial resolution of the following structures: round and oval windows, incudomallear and incudostapedial joints, basal turn spiral lamina, and scutum. The paired z-axis ultra-high-resolution and ultra-high-resolution-iterative reconstruction images were displayed side by side in random order, with readers blinded to the imaging protocol. Image noise was compared in ROls over the posterior fossa.

RESULTS: We identified 8 patients, yielding 16 sets of temporal bone images (left and right). Three sets were excluded because the patient underwent surgery between the 2 examinations. Spatial resolution was comparable (Poschl) or slightly better (axial and coronal planes) with ultra-high-resolution-iterative reconstruction than with z-axis ultra-high-resolution. A paired $t$ test indicated that noise was significantly lower with ultra-high-resolution-iterative reconstruction than with z-axis ultra-high-resolution $(P<.001)$, with a mean noise reduction of $37 \%$ (range, $18 \%-49 \%$ ).

CONCLUSIONS: The ultra-high-resolution-iterative reconstruction scan mode has similar or slightly better resolution relative to the z-axis ultra-high-resolution mode for CT of the temporal bone but significantly $(P<.01)$ lower image noise, which may enable the dose to be reduced by approximately $50 \%$.

ABBREVIATIONS: IR = iterative reconstruction; $U H R=$ ultra-high-resolution; $z U H R=$ z-axis ultra-high-resolution

$\mathbf{S}^{\mathrm{i}}$ ince the introduction of multidetector techniques, CT has become a major diagnostic technique for temporal bone imaging because its high spatial resolution is well-suited to the task of visualizing the fine anatomic structures of the middle and inner ear. ${ }^{1-5}$ To improve spatial resolution, different approaches have been introduced. One of these is the use of an attenuating comb filter to reduce the detector aperture in both fan and cone angle directions, which is referred to as the z-axis ultra-high-resolution

Received November 3, 2014; accepted after revision February 16, 2015

From the Departments of Radiology (S.L., F.E.D., J.I.L, K.K.K., R.J.W., C.H.M.) and Health Sciences Research, Division of Biomedical Statistics and Informatics (R.E.C.) Mayo Clinic, Rochester, Minnesota.

Paper previously presented at: Annual Meeting of the Radiological Society of North American, December 1-6, 2013; Chicago, Illinois.

Please address correspondence to Shuai Leng, PhD, Department of Radiology, Mayo Clinic, 200 First St SW, Rochester, MN 55905; e-mail:leng.shuai@mayo.edu

http://dx.doi.org/10.3174/ajnr.A4338
(zUHR) technique. ${ }^{6}$ This technique, in combination with a flying focal spot technique, provides nominal image thickness thinner than the detector cell size at the isocenter. ${ }^{6,7}$

Due to the requirement for high spatial resolution, the radiation dose in temporal CT can be high, especially with the zUHR technique because its dose efficiency is reduced as photons passing through the patient are blocked from the detector by the comb filters in both fan and cone angle directions. ${ }^{8} \mathrm{~A}$ recent focus of CT imaging has been to reduce patient exposure to ionizing radiation, following the as low as reasonably achievable principle. ${ }^{9-13}$ However, the consequent reduction in photons can adversely affect image quality and present a great challenge when imaging small, anatomically complex structures embedded in attenuating bone, such as those of the middle and inner ear. Iterative reconstruction (IR) is a promising reconstruction technique that is superior to standard filtered back-projection reconstructions and theoretically can be 
used to improve resolution at standard radiation doses or to maintain current resolution by using a reduced radiation dose. ${ }^{14-17}$

Recently, a new technique combining a deconvolution technique and an IR algorithm, referred to as ultra-high-resolution (UHR)-IR, has been introduced to improve dose efficiency of the zUHR mode. Phantom studies demonstrated that this technique improved dose efficiency by removing the comb filter along the cone $(\mathrm{z})$ direction. ${ }^{8}$ In this study, we retrospectively reviewed temporal bone CT examinations in patients who had baseline studies by using the standard zUHR technique and follow-up examinations by using UHR-IR to determine whether UHR-IR provided improved resolution and lower noise than zUHR in the clinical setting, which could enable reductions in dose.

\section{MATERIALS AND METHODS}

\section{Patient Enrollment and CT Scans}

This retrospective study was approved by our institutional review board and was Health Insurance Portability and Accountability Act-compliant. Patients with temporal bone CT scans acquired by using a zUHR protocol who underwent a follow-up scan by using the UHR-IR technique were identified by searching the electronic medical records. Patients who had not provided authorization for research were excluded from this study. Temporal bones in which inner ear surgery was performed between the 2 examinations were also excluded.

Baseline scans were acquired on a 64-section CT scanner (Sensation 64; Siemens, Forchheim, Germany) by using the zUHR mode $(12 \times 0.3 \mathrm{~mm}$ collimation), with a tube potential of $120 \mathrm{kV}$, 400 effective mAs, 1 -second rotation time, and 0.8 helical pitch. The automatic exposure control was off, and the volume CT dose index was $88 \mathrm{mGy}$. Images were reconstructed by using a standard filtered back-projection algorithm with a special kernel designed for the UHR mode (U70). Images were reconstructed with $0.4-\mathrm{mm}$ section thickness at $0.3-\mathrm{mm}$ increments. Both the $\mathrm{z}$-axis and in-plane flying focal spot were used for data acquisition. ${ }^{6,7}$

The follow-up UHR-IR scans were conducted on a 128-section CT scanner (Somatom Definition Flash; Siemens) by using the UHR scan mode $(16 \times 0.6 \mathrm{~mm}$ collimation), with a tube potential of $120 \mathrm{kV}, 375$ effective mAs, 1-second rotation time, and 0.8 helical pitch. The automatic exposure control was off, and the volume CT dose index was $82 \mathrm{mGy}$. Images were reconstructed by using an IR algorithm (sinogram-affirmed iterative reconstruction, SAFIRE; Siemens) with a special kernel designed for the UHR mode (V80). The strength of the IR algorithm was set at 3 on a scale of 1 (least noise reduction) to 5 (most noise reduction). The thinnest available image section thickness $(0.5 \mathrm{~mm})$ was used, with an increment of $0.3 \mathrm{~mm}$.

For both original and follow-up examinations, images of the left and right temporal bones were reconstructed in the axial, coronal, and Poschl planes, as per our routine clinical protocol.

\section{Assessment of Spatial Resolution}

Image quality was independently assessed by 3 fellowship-trained neuroradiologists experienced in temporal bone image interpretation, with a focus on differences in image sharpness (spatial resolution) between the 2 techniques. All images were reviewed

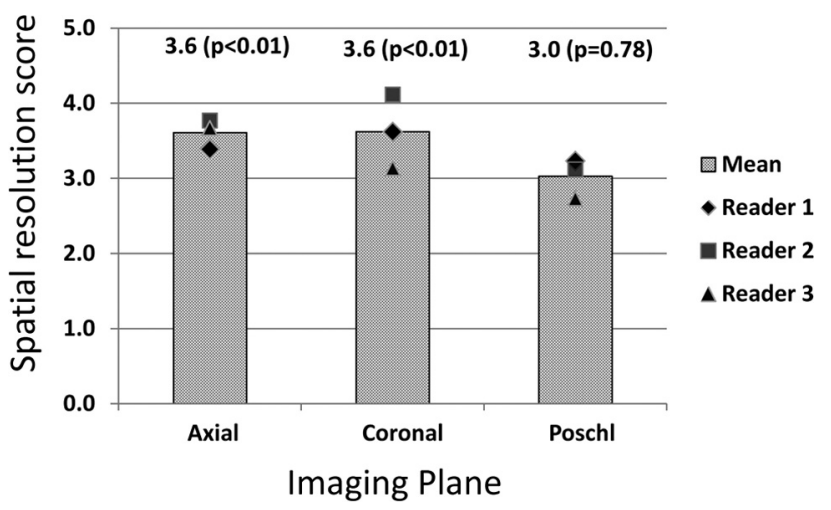

FIG 1. Spatial resolution scores for images in the axial, coronal, and Poschl planes, averaged across individual structures in each imaging plane. The scale assessed UHR-IR images relative to zUHR images: $1=$ inferior resolution with degraded visualization, 2 = slightly inferior resolution without affecting visualization; $3=$ equivalent, $4=$ slightly superior resolution without affecting visualization, 5 = superior resolution with improved visualization. The means are shown as lined bars, with the value above each bar. Statistical significance was determined with the Wilcoxon signed rank test.

on a calibrated monitor used for clinical diagnosis located inside a darkened room, with ambient light $<10$ lux. Baseline and follow-up images of the same patient and same side of the head in the 3 planes were displayed side by side in a randomized order, with the readers blinded to reconstruction parameters. The 3 neuroradiologist reviewers assessed the spatial resolution on each of the axial, coronal, and Poschl planes, focusing on the following structures: round window, incudomallear joint, and basal turn spiral lamina (axial plane); oval window and scutum (coronal plane); and the basal turn spiral lamina and incudostapedial joint (Poschl plane). Readers compared the spatial resolution of the displayed image on the left with that displayed on the right, and another investigator (not a reader) determined post hoc which image was zUHR and which was UHR-IR to apply the following grading scale to the UHR-IR images (relative to the zUHR images) for each structure: $1=$ inferior resolution with degraded visualization, 2 = slightly inferior resolution without affecting visualization, 3 = equivalent, $4=$ slightly superior resolution without affecting visualization, $5=$ superior resolution with improved visualization.

\section{Image Noise Measurement}

Image noise was measured as the $\mathrm{SD}$ of $\mathrm{CT}$ numbers inside a circular ROI placed on the axial images. The ROI size was approximately $0.4 \mathrm{~cm}^{2}$ and was placed over the posterior fossa area, with locations matched as closely as possible between those of the zUHR and UHR-IR scans.

\section{Statistical Analysis}

The Wilcoxon signed rank test was performed to compare the scores of image resolution between UHR-IR and zUHR for the aforementioned individual structures (round window, incudomallear joint, oval window, incudostapedial joint, spiral lamina in the basal turn, and scutum) and reconstruction planes (axial, coronal, and Poschl). For the comparison of spatial resolution in reconstruction planes, the averaged score of structures in the plane was used. A 2-tailed paired $t$ test was used to compare image 
Spatial resolution scores and $\boldsymbol{P}$ values

\begin{tabular}{lcccccc}
\hline & $\begin{array}{c}\text { Round } \\
\text { Window }\end{array}$ & $\begin{array}{c}\text { Incudomallear } \\
\text { Joint }\end{array}$ & $\begin{array}{c}\text { Basal Turn } \\
\text { Spiral Lamina }\end{array}$ & $\begin{array}{c}\text { Oval } \\
\text { Window }\end{array}$ & Scutum & $\begin{array}{c}\text { Incudostapedial } \\
\text { Joint }\end{array}$ \\
\hline Mean score & 4.05 & 4.05 & 2.69 & 3.71 & 3.53 & 3.38 \\
$P$ value & $<.001$ & $<.001$ & .0083 & .0011 & .0037 & .0248 \\
\hline
\end{tabular}

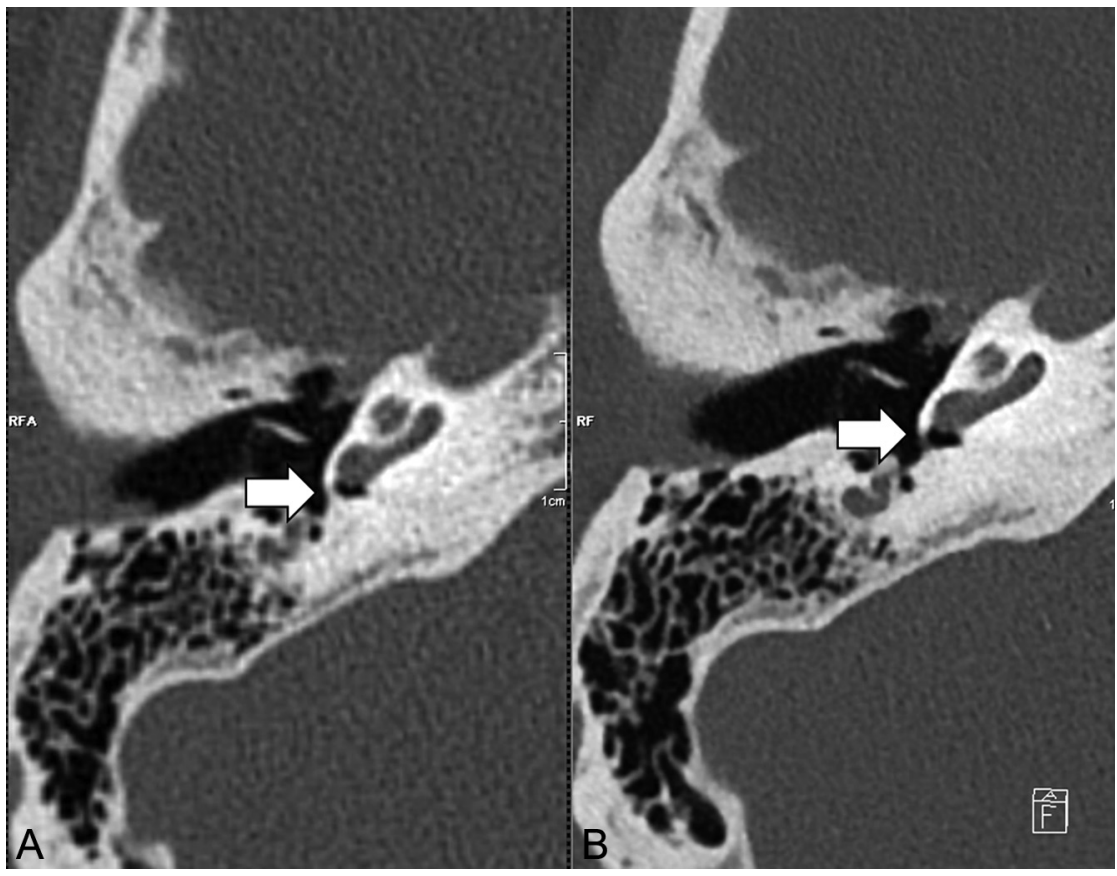

FIG 2. Comparison of spatial resolution of the round window. Representative axial CT images of the round window of the same patient scanned with the zUHR technique $(A)$ and UHR-IR technique (B). The UHR-IR technique produced superior spatial resolution and lower image noise.

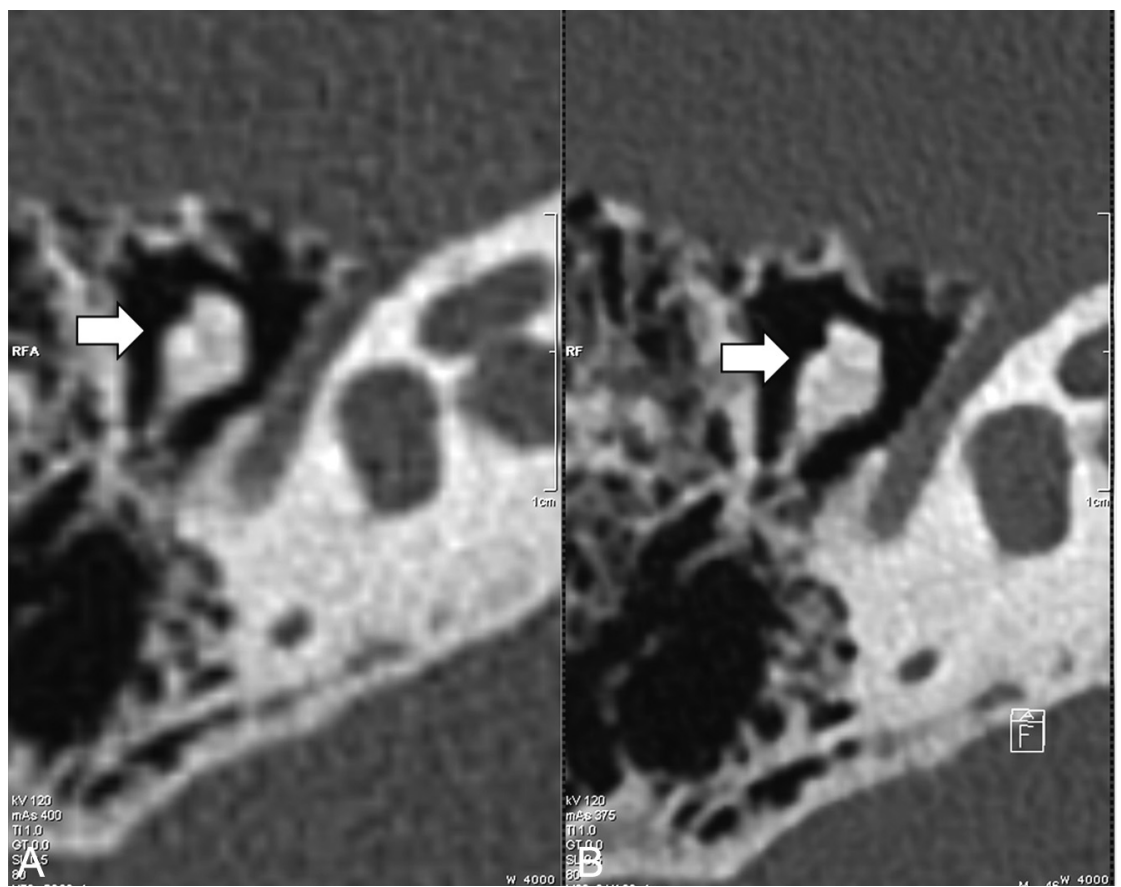

FIG 3. Comparison of the spatial resolution of the incudomallear joint. Representative axial images of the incudomallear joint of the same patient scanned with the zUHR technique $(A)$ and UHR-IR technique $(B)$. The UHR-IR technique produced superior spatial resolution and lower image noise. noise between the 2 techniques. With both tests, $P<.01$ was considered a statistically significant difference. The difference in image noise between the 2 techniques was calculated, and dose reduction was estimated on the basis of the relationship between image noise and radiation dose (ie, radiation dose is inversely proportional to the square of image noise in CT).

\section{RESULTS}

\section{Study Sample}

We identified 8 patients ( 2 male and 6 female; age ranges, 16-75 years of age) who had initial examinations with the zUHR technique and underwent a follow-up scan by using the new UHR-IR technique. Images from the left and right side of each patient provided 16 sets of temporal bone CT images. Three of these datasets were excluded because the patient had inner ear surgery between the 2 examinations; this change left 13 sets of images included in the final data analysis. The median time interval between examinations was 12 months (range, 1-34 months). For the initial examinations, the principal indications were the following: hearing loss $(n=4 ; 2$ conductive and 2 sensorineural), inflammatory disease $(n=2)$, trauma $(n=1)$, and skeletal dysplasia $(n=1)$. For the follow-up examinations, the indications were the following: postoperative evaluation $(n=3 ; 1$ internal auditory canal decompression, 1 cochlear implant, and 1 ossicular reconstruction), follow-up or ruling out inflammatory disease $(n=3)$, further evaluation of bilateral fractures $(n=1)$, and follow-up of a presumed mastoid hemangioma $(n=1)$.

\section{Spatial Resolution}

Spatial resolution scores showed that the 3 readers found the UHR-IR images to be of significantly higher quality $(P<.01$, Wilcoxon signed rank test) than the zUHR images in the axial and coronal planes but not in the Poschl plane (Fig 1). Spatial resolution scores of individual structures showed that readers found the UHR-IR images to be of significantly higher quality $(P<.01$, Wilcoxon signed rank test) for the round window, incudomallear joint, oval window and scutum (Table). UHR images had 
higher quality than zUHR images for the incudostapedial joint, but the difference was not statistically significant (Table). For the basal turn spiral lamina, UHR images had a lower quality than zUHR images (Table). The superior sharpness produced by the UHR-IR technique can be seen in representative images at the round window and incudomallear joint (Figs 2 and 3).

\section{Noise and Potential Dose Reduction}

In all cases, images obtained with UHR-IR had lower noise than those obtained with zUHR (all, $P<.01$; paired $t$ test; Fig 4 ). In representative images (Fig 5), the UHR-IR and zUHR techniques produced similar sharpness, but the UHR-IR images had much

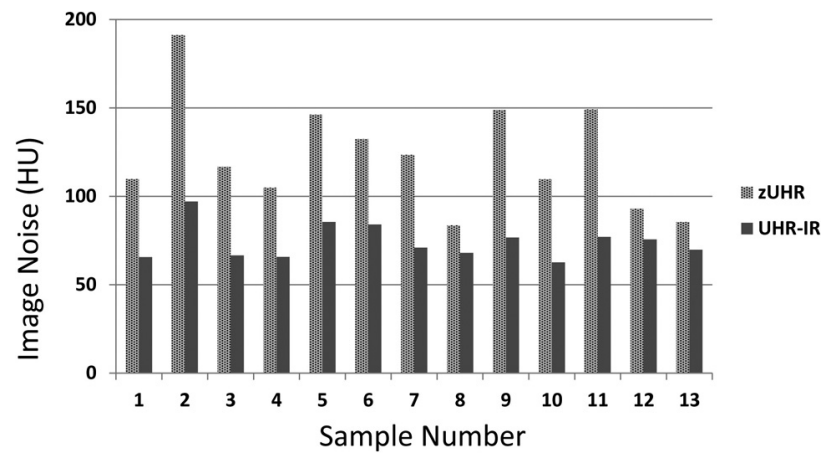

FIG 4. Lower image noise in images acquired with UHR-IR. Image noise was measured at the posterior fossa in axial images from each of the 13 datasets by using the zUHR and UHR-IR techniques.

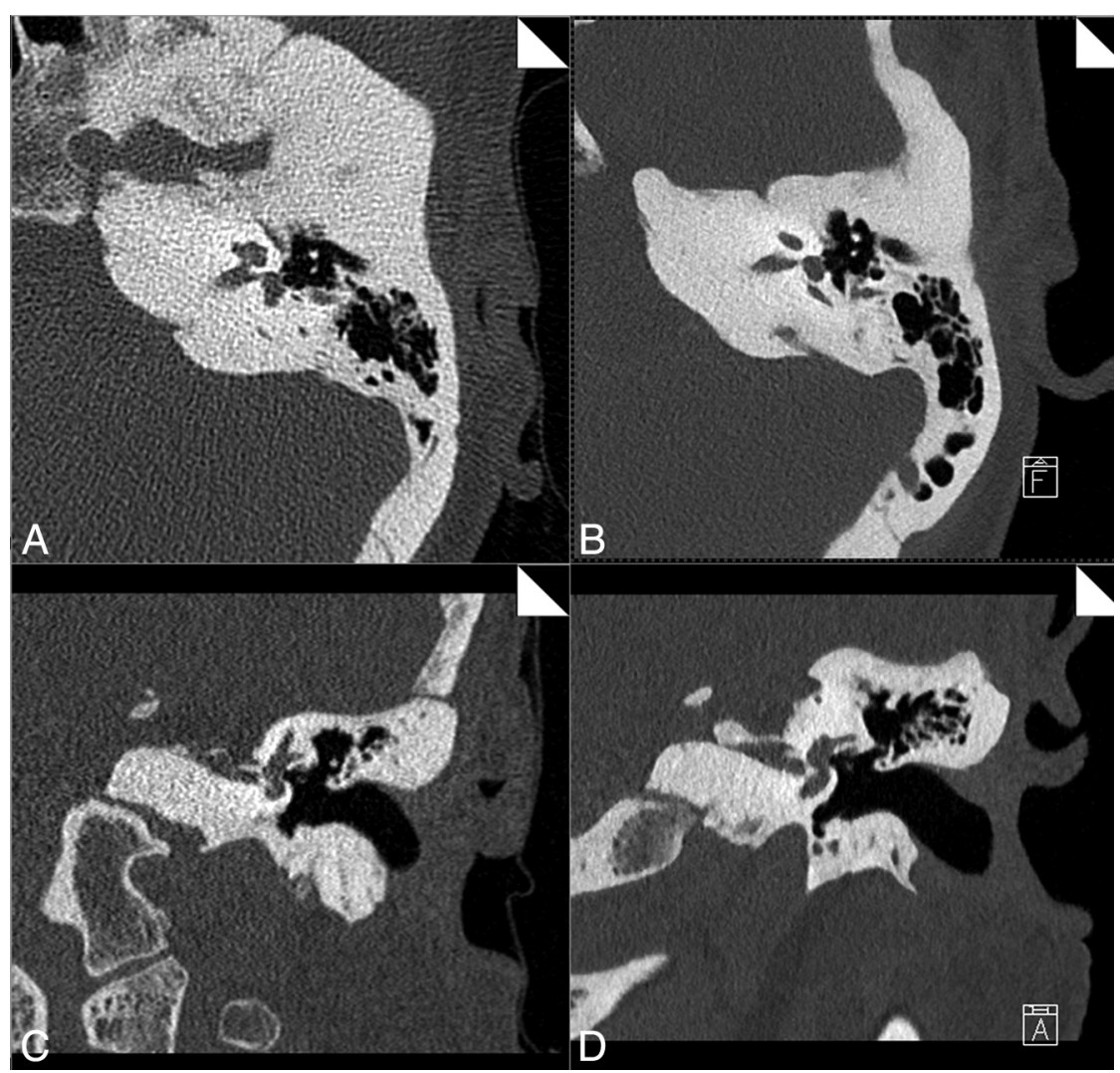

FIG 5. Spatial resolution and image noise in images from a representative patient. Axial $(A$ and $B)$ and coronal $(C$ and $D) C T$ images of the same patient scanned with the zUHR technique $(A$ and $C)$ and UHR-IR technique $(B$ and $D)$. Substantial noise reduction was achieved by using the UHR-IR technique. lower image noise in both the axial and coronal planes. The mean noise reduction by using UHR-IR, relative to $\mathrm{zUHR}$, was $37 \%$ (range, 18\%-49\%). This translated to a dose reduction potential of $61 \%$ (range, $33 \%-74 \%$ ).

\section{DISCUSSION}

Reduction in the ionizing radiation dose has been a major focus of technology development in diagnostic CT in recent years..$^{9-11,14,18}$ Temporal bone CT requires a high radiation dose relative to other diagnostic CT examinations; for example, temporal bone CT by using the standard technique at our institution (zUHR) results in a volume CT dose index of $88 \mathrm{mGy}$, compared with 38-69 mGy for a routine head CT examination. Given that radiation reduction can adversely affect image quality, techniques that allow dose reduction without sacrificing image resolution would be exceedingly useful in this area of CT imaging.

In this study, radiation dose reduction was achieved by using 2 techniques: 1) the deconvolution technique, and 2) iterative reconstruction. The deconvolution technique allows removal of the comb filter along the z-direction compared with zUHR, which substantially improves the dose efficiency by increasing the number of photons detected by the detector. Most important, the spatial resolution was preserved, which was substantially better than that in other scanners without the comb filter techniques. ${ }^{6}$ Iterative reconstruction has the potential to reduce image noise and radiation dose compared with standard filtered back-projection reconstruction algorithms in many studies, ${ }^{14-17}$ including in the temporal bone. ${ }^{13}$ The amount of dose reduction highly depends on the specific vendor, scanner platform and imaging task. Dose reduction in this study was a combination of these 2 techniques, similar to that demonstrated in the previous phantom studies by comparing filtered back-projection-zUHR, IR-zUHR, and IR-UHR. ${ }^{8}$

It is important to maintain the spatial resolution while reducing image noise and radiation dose, especially in temporal bone CT, in which high spatial resolution is critical. In this study, we found that the UHR-IR scan mode by using a z-deconvolution technique produced resolutions similar to or slightly better than those produced by the zUHR mode, but with significantly (37\%) lower image noise. This reduced image noise could potentially allow UHR-IR to be used to reduce the dose by $>50 \%$ on the basis of the relationship between image noise and radiation dose in CT. This study was performed by using the IRUHR technique on the second-generation dual-source scanner (Flash) and compared to the same patients' prior examinations on older scanners. By comparing scans from the same patients, we avoided the potential compounding fac- 
tor caused by patient-to-patient variation. The IR-UHR technique has also been implemented on the third-generation dualsource scanner, and dose reduction was reported by comparing scans of different patients randomly assigned to the first-, second-, and third-generation dual-source scanners. ${ }^{19}$ Given the improved source and detector technologies, more dose reduction may be achievable by using the third-generation dual-source scanner.

There are several limitations to this study. The first is the small patient cohort, due to the limited number of patients scanned with both zUHR and UHR-IR. However, image noise was lower with UHR-IR for each individual case, and the paired $t$ test showed this difference to be statistically different. Another limitation is that the retrospective methodology did not permit standardization of the time interval between examinations. The third limitation of this study is that the zUHR examinations were performed on a different scanner platform from that of the UHR-IR examinations. This was because prior examinations were only available on a different scanner platform and because the new scanner was only recently available. The amount of dose reduction could be potentially less if the zUHR examinations were performed on the same new scanner platform.

\section{CONCLUSIONS}

This study demonstrated that UHR-IR reduces image noise by $>30 \%$ while providing similar or better spatial resolution than the existing zUHR technique. This may enable a substantial reduction in radiation dose without a corresponding loss of resolution. This is a considerable achievement for temporal bone CT, which currently requires one of the highest doses in clinical CT imaging. These preliminary findings need to be corroborated with further studies performed with a reduced radiation dose.

\section{ACKNOWLEDGMENTS}

The authors thank Thomas Vrieze, Mike Bruesewitz, Sally Reinhart, and Christine Tipka for helping collect patient data and Kristina Nunez and Naomi Ruff for their assistance in manuscript preparation.

Disclosures: Cynthia H. McCollough-UNRELATED: Grants/Grants Pending: Siemens, ${ }^{*}$ Comments: Dr McCollough reports grants from Siemens, outside the submitted work. *Money paid to the institution.

\section{REFERENCES}

1. Lane JI, Lindell EP, Witte RJ, et al. Middle and inner ear: improved depiction with multiplanar reconstruction of volumetric CT data. Radiographics 2006;26:115-24
2. Lane JI, Witte RJ. Temporal Bone: An Imaging Atlas. Berlin: SpringerVerlag; 2009

3. Purcell DD, Fischbein NJ, Patel A, et al. Two temporal bone computed tomography measurements increase recognition of malformations and predict sensorineural hearing loss. Laryngoscope 2006;116:1439-46

4. Swartz JD, Loevner LA. Imaging of the Temporal Bone. New York: Thieme Medical; 2008

5. Noble JH, Dawant BM, Warren FM, et al. Automatic identification and $3 \mathrm{D}$ rendering of temporal bone anatomy. Otol Neurotol 2009;30:436-42

6. Flohr T, Stierstorfer K, Süß C, et al. Novel ultrahigh resolution data acquisition and image reconstruction for multi-detector row $\mathrm{CT}$. Med Phys 2007;34:1712

7. Flohr TG, Stierstorfer K, Ulzheimer S, et al. Image reconstruction and image quality evaluation for a 64-slice CT scanner with z-flying focal spot. Med Phys 2005;32:2536-47

8. McCollough CH, Leng S, Sunnegardh J, et al. Spatial resolution improvement and dose reduction potential for inner ear CT imaging using a z-axis deconvolution technique. Med Phys 2013;40:061904

9. McCollough C, Chen G, Kalender WA, et al. Achieving routine submSv CT scanning: report from the summit on management of radiation dose in CT. Radiology 2012;264:567-80

10. McCollough CH, Primak AN, Braun N, et al. Strategies for reducing radiation dose in CT (PMC 2743386). Radiol Clin North Am 2009;47:27-40

11. Yu L, Liu X, Leng S, et al. Radiation dose reduction in CT: techniques and future perspective. Imaging Med 2009;1:65-84

12. Nauer C, Rieke A, Zubler C, et al. Low-dose temporal bone CT in infants and young children: effective dose and image quality. AJNR Am J Neuroradiol 2011;32:1375-80

13. Niu Y, Mehta D, Zhang Z, et al. Radiation dose reduction in temporal bone CT with iterative reconstruction technique. AJNR Am J Neuroradiol 2012;33:1020-26

14. Thibault JB, Sauer KD, Bouman CA, et al. A three-dimensional statistical approach to improved image quality for multislice helical CT. Med Phys 2007;34:4526-44

15. Winklehner A, Karlo C, Puippe G, et al. Raw data-based iterative reconstruction in body CTA: evaluation of radiation dose saving potential. Eur Radiol 2011;21:2521-26

16. Singh S, Kalra MK, Hsieh J, et al. Abdominal CT: comparison of adaptive statistical iterative and filtered back projection reconstruction techniques. Radiology 2010;257:373-83

17. Silva AC, Lawder HJ, Hara A, et al. Innovations in CT dose reduction strategy: application of the adaptive statistical iterative reconstruction algorithm. AJR Am J Roentgenol 2010;194:191-99

18. National Council on Radiation Protection and Measurements. Ionizing radiation exposure of the population of the United States. Bethesda: National Council on Radiation Protection and Measurements; 2009: Report 160

19. Meyer M, Haubenreisser $H$, Raupach $\mathrm{R}$, et al. Initial results of a new generation dual source CT system using only an in-plane comb filter for ultra-high resolution temporal bone imaging. Eur Radiol 2015;25:178-85 